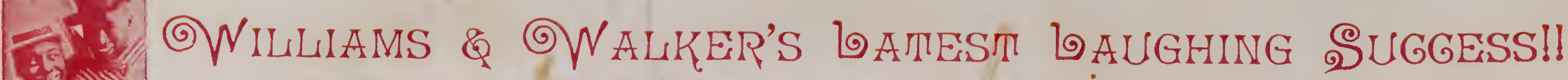
Introduced in their New Playu"TIEF POIIOY: SIIOP."

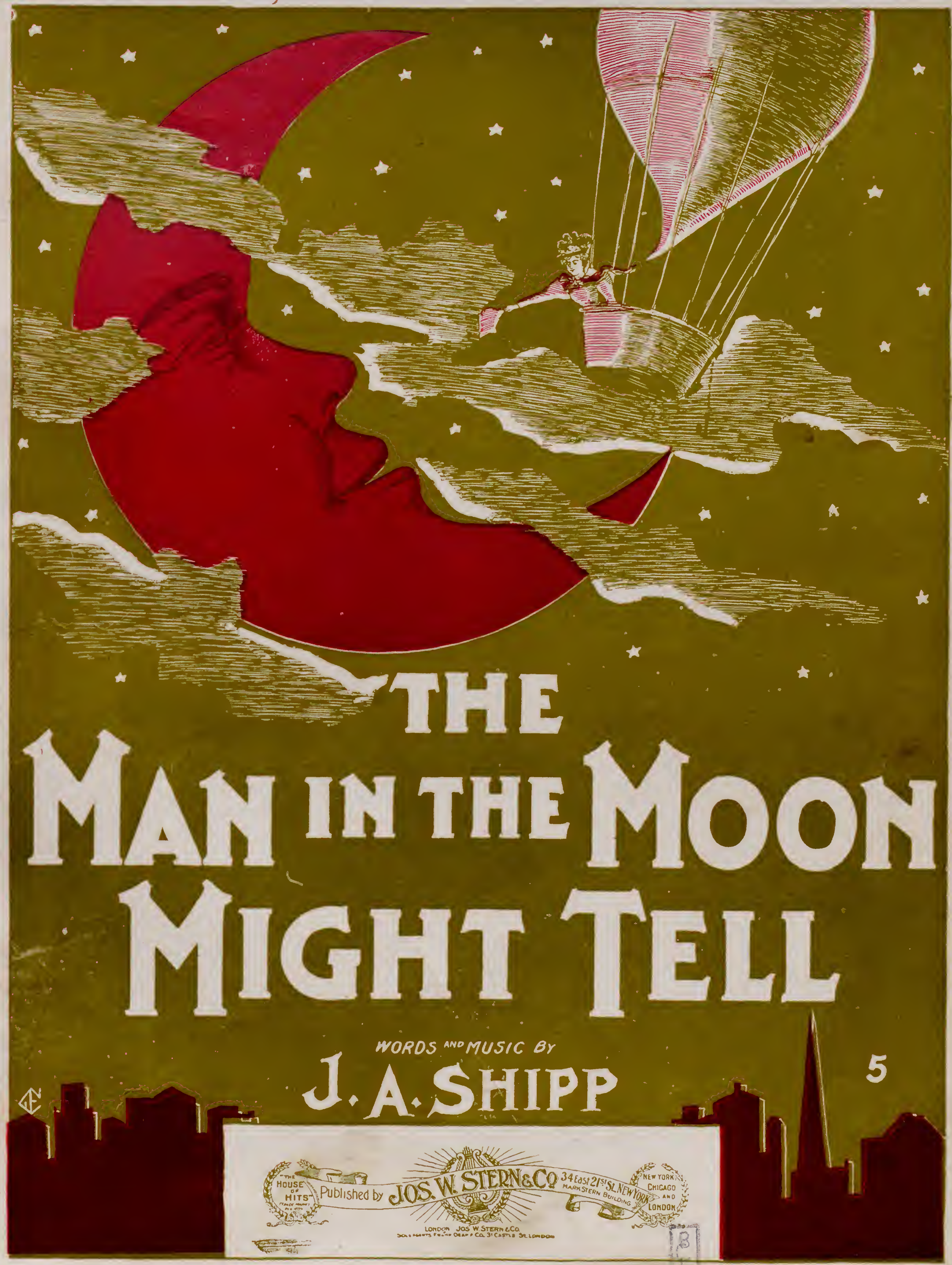


$Y-\cdots$

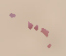




\section{THE MAN IN THE MOON MIGHT TELL.}

Words \& Music by J. A. Shipp.
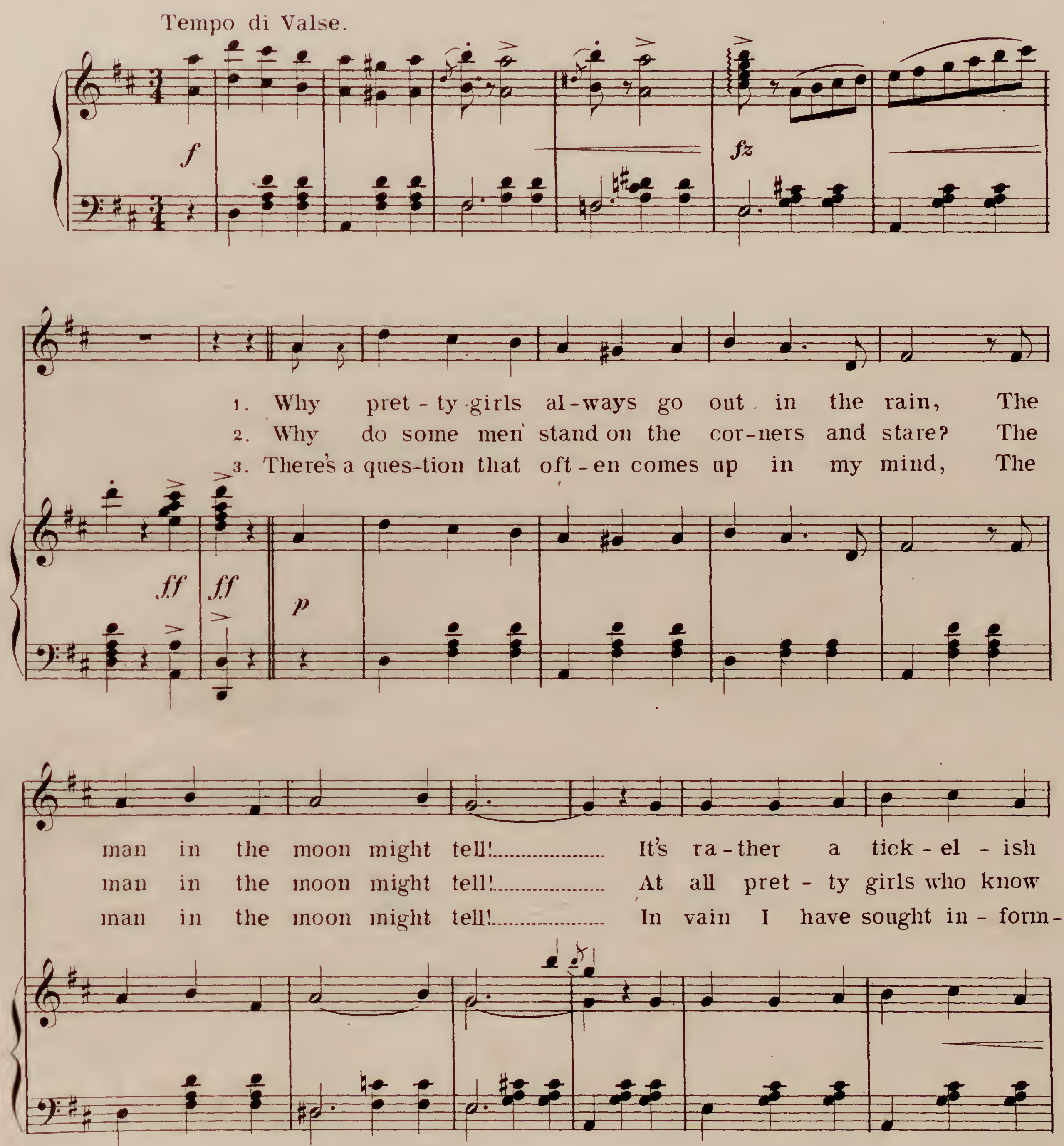

Copyright 18.9 .9 by Jos. W. Stern \& Co.

Copyright and performing rights secured for Great Britain and all British Colonies and. Possessions.

2011-3 Reproduction of this Music on Mechanical Instruments strictly prohibited. All rights reserved. 
4
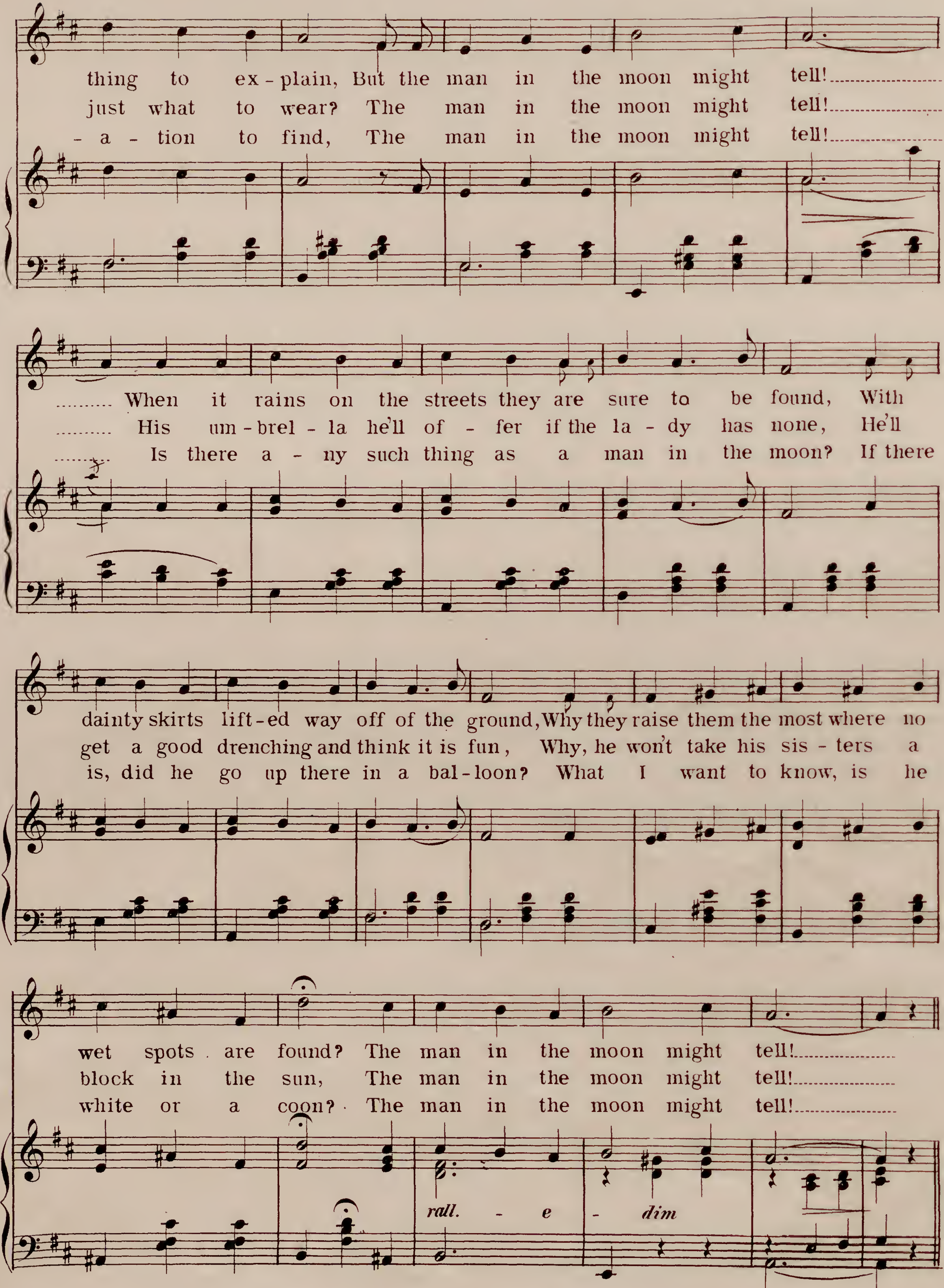

2011-3 
CHORUS.
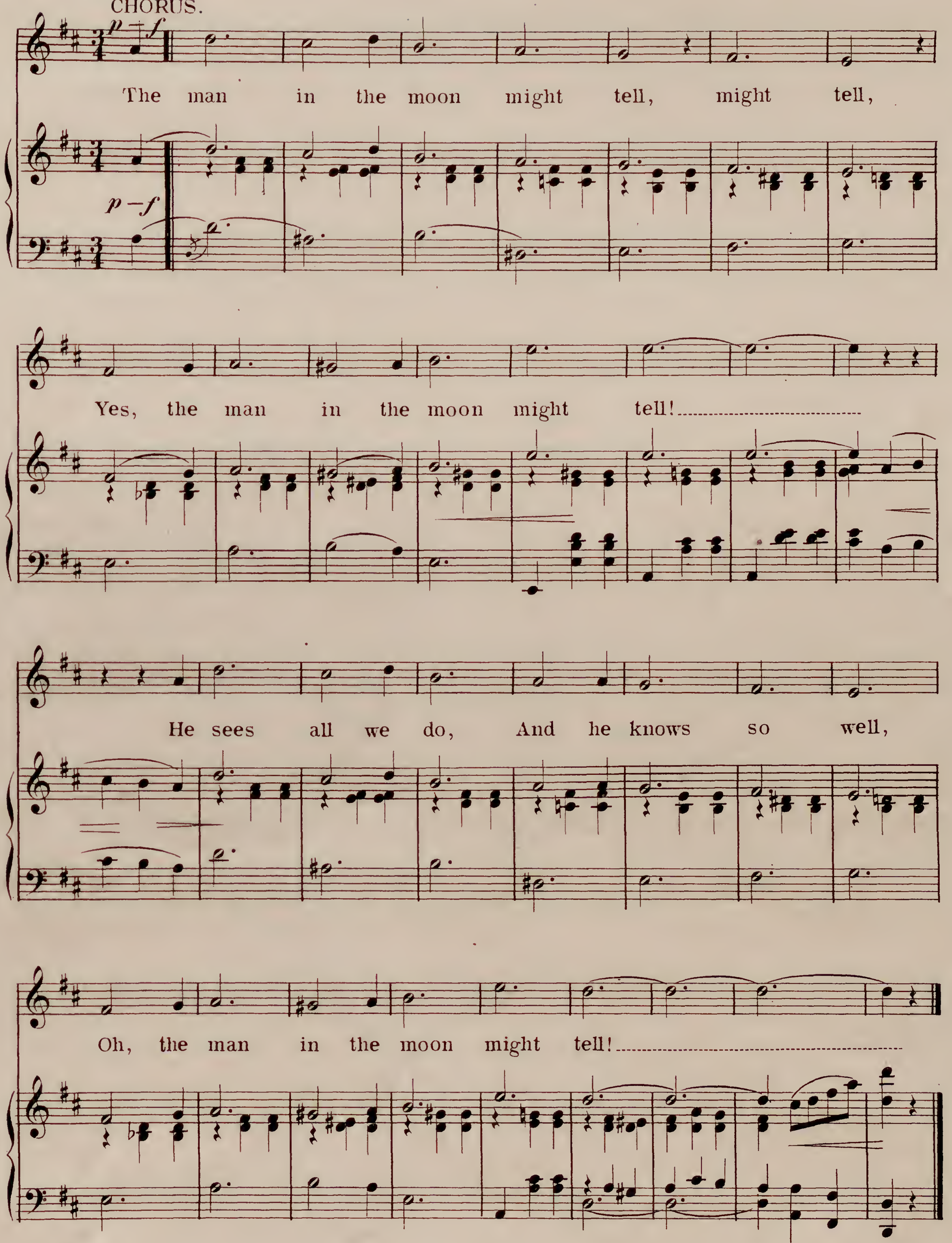

2011-3 


\section{Popular Instrumental Compositions by Well Known Composers.}

Try them over on your piano.

* Espanita" Waltzes. Geo. ROSEY. Piano Solo.60 Orchestra.75 Band 2.00

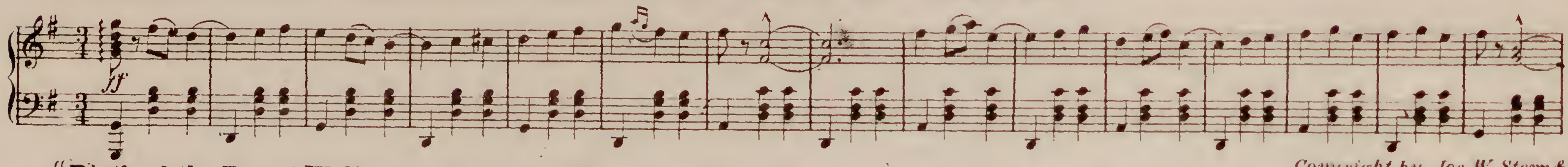

"Birth of the Rose" Waltzes. MAX S. WITT. Piano Solo .50 orchestra .5.5

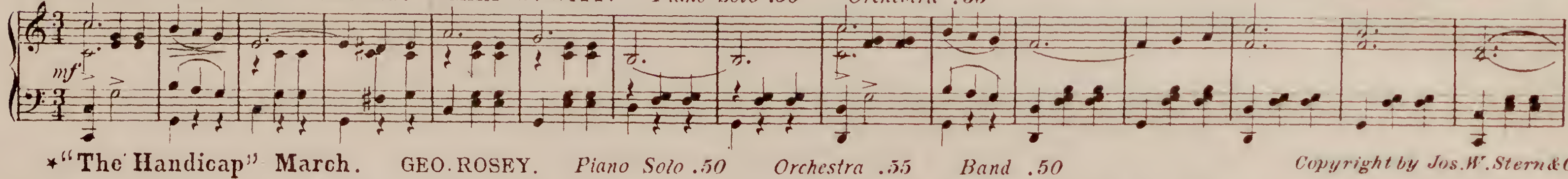

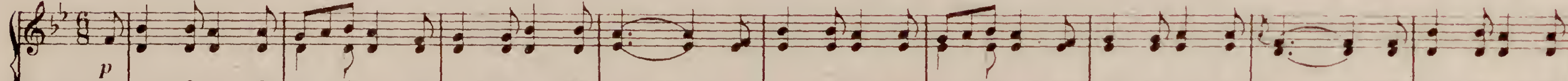

"Eg

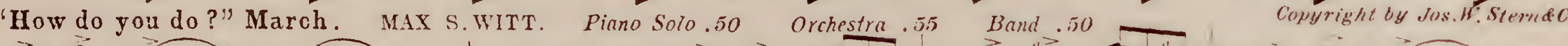

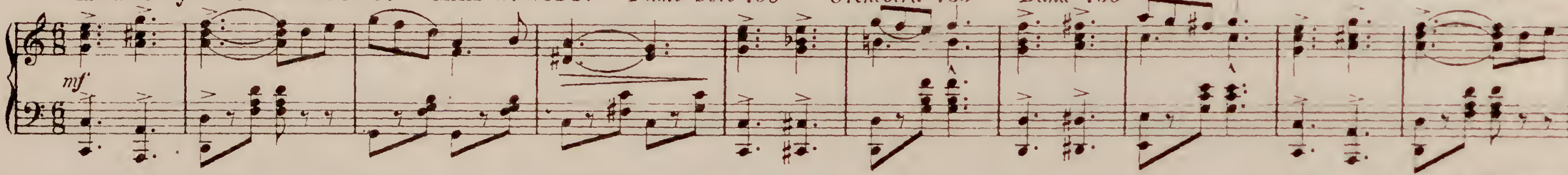

"Pickaninny's Screnade" Polka. R. ARoxson. Pino Solo.jo Orchestra .55

Copyright by Jos. H. Sternde.

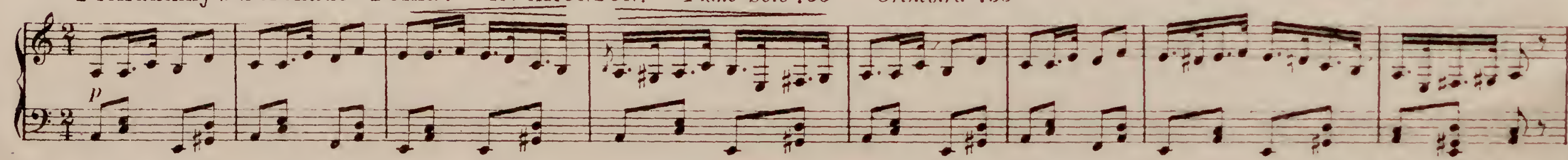

* "Swoet Reposo" Waltzes. MAX S.WITT. Piano Solo.50 Orchestra .75 . Band 1.50

Copyright by ons. It. Sterndro.

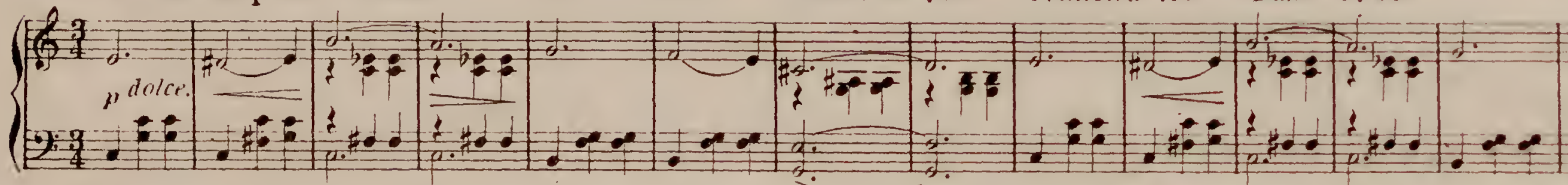

* "The Rainbow Dance" Characteristic. GEO. ROSEY. Piano Solo.50 Orchestra .j5, Rand .50

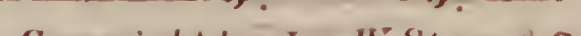

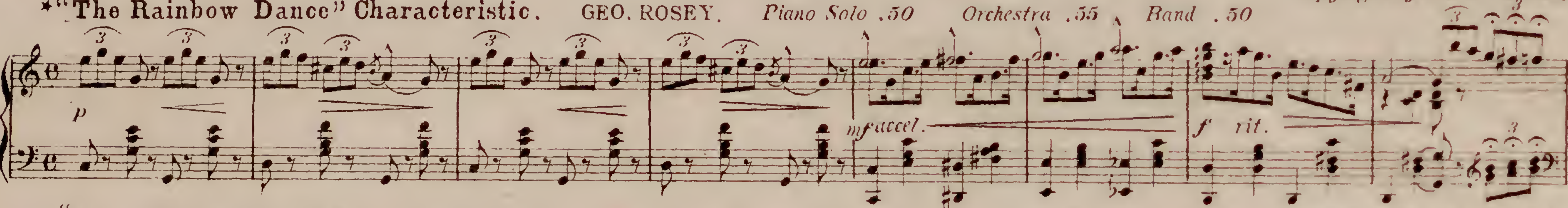

* "King Carnival" March. GEO.ROSEY. Piano Solo.50 Orchestra .5.) Band .50

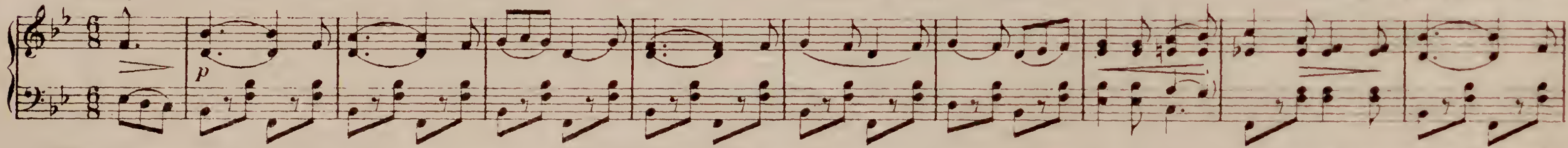

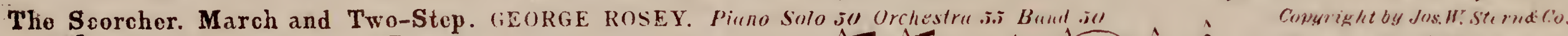

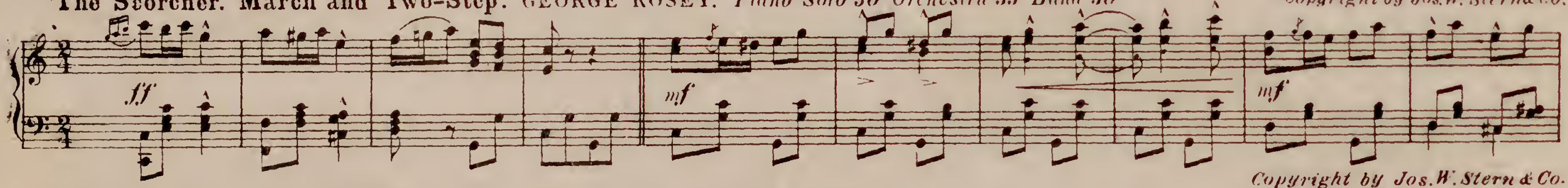

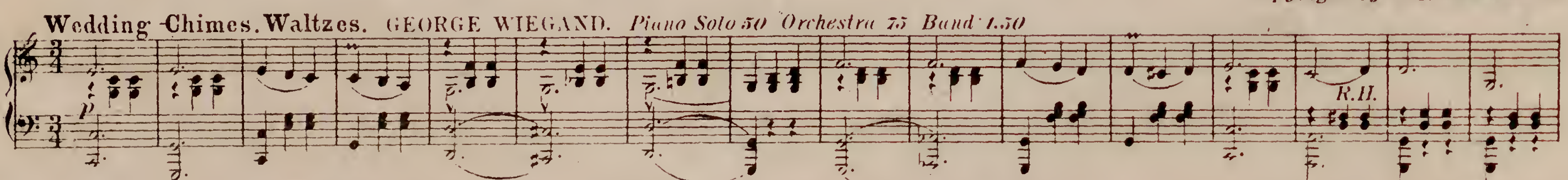

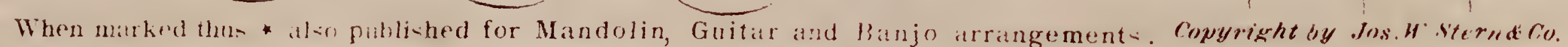
Full and Complete Copies for sale at all Musir Stores.

Jos. W. Stern and Co, Publishers. 34 East 21: Street, New-York. 

New and Beautiful Waltzes Grand and Inspiring Marches by the famous GEORGE ROSEY and other composers.

330. ROSETS NIW PATBIOTIC MASTERTIRCB TEIE SEITIT OF IIBERTY

a Susana.

Spanish Waltzes.

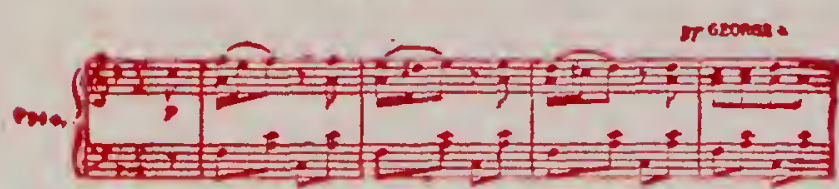

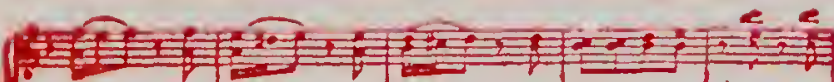

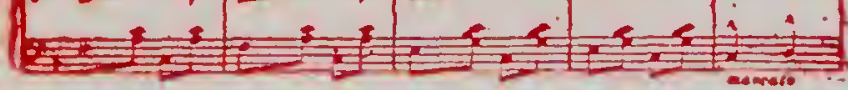

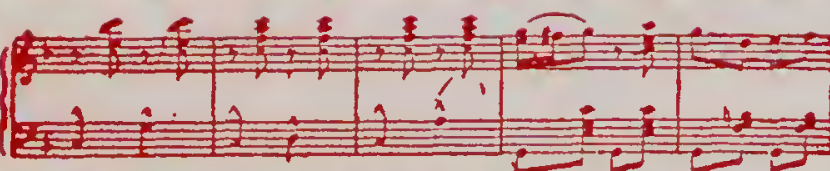

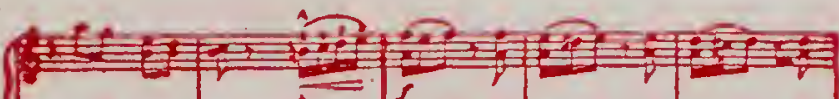

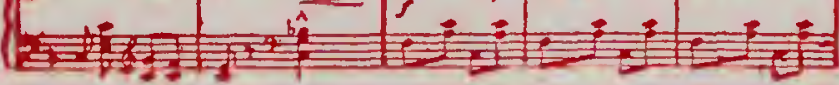

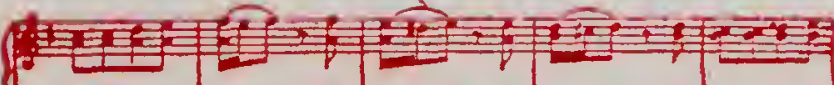

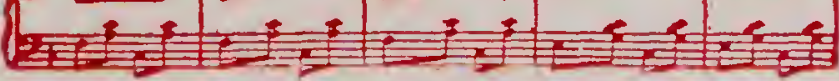

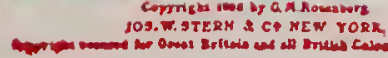

The Hottest Two-Step.... SLNCE HLS PAMOUS "HONEYHOOS. -

The Scorcher

March and Two.Step
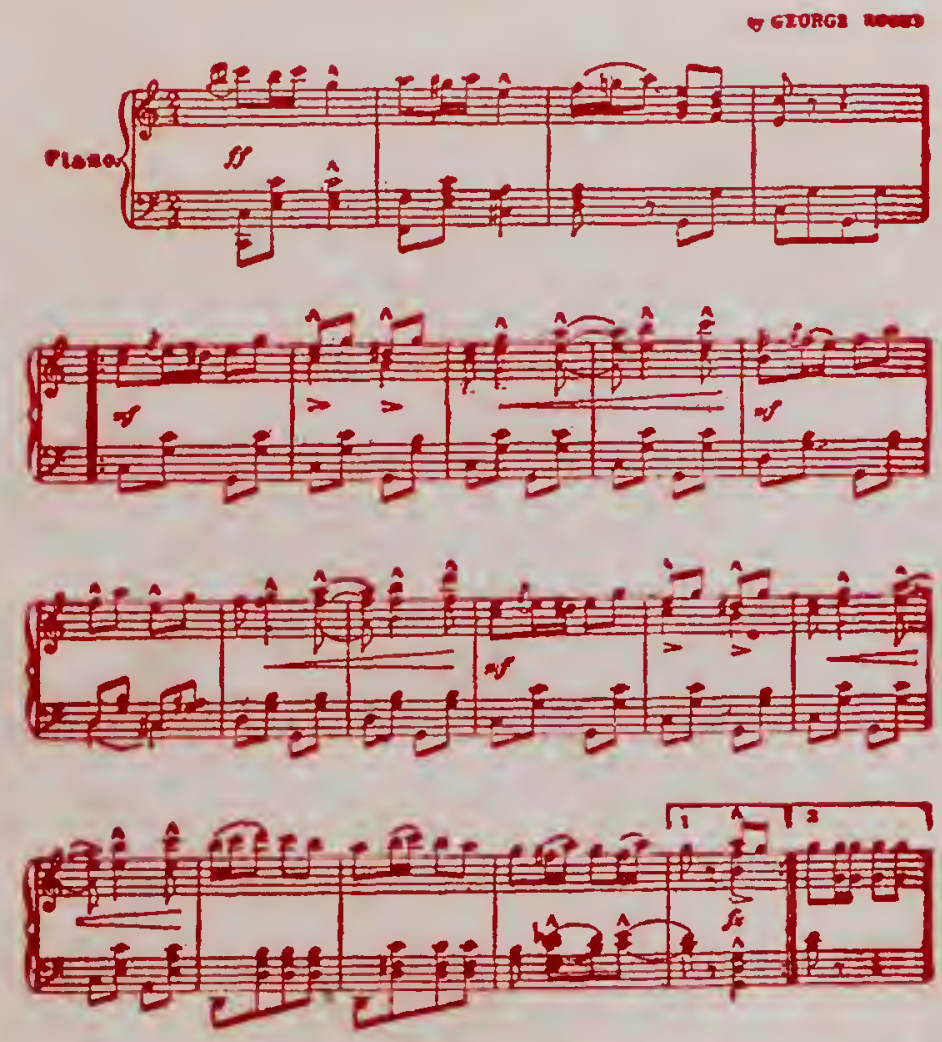

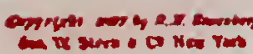

HOT TIME LN MOBILE ARCB TWO STEP O CABEWALE

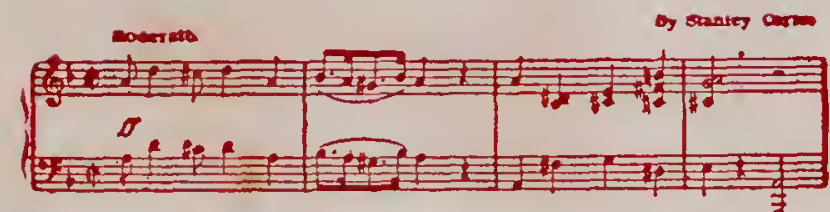

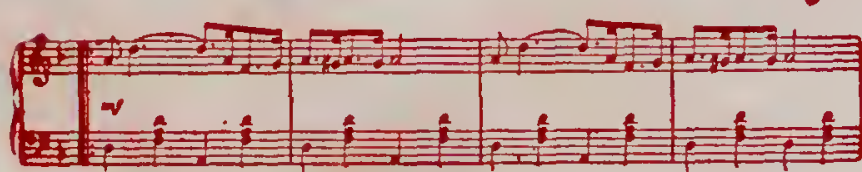

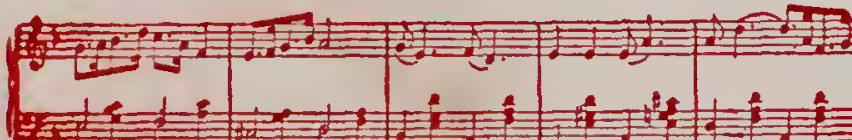

(1)

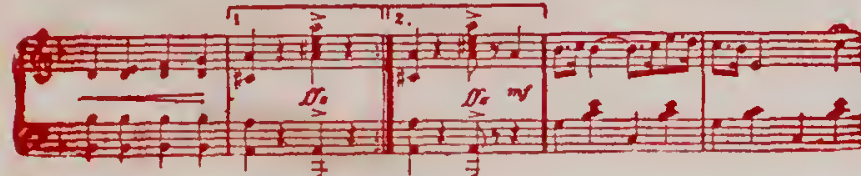

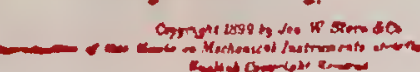

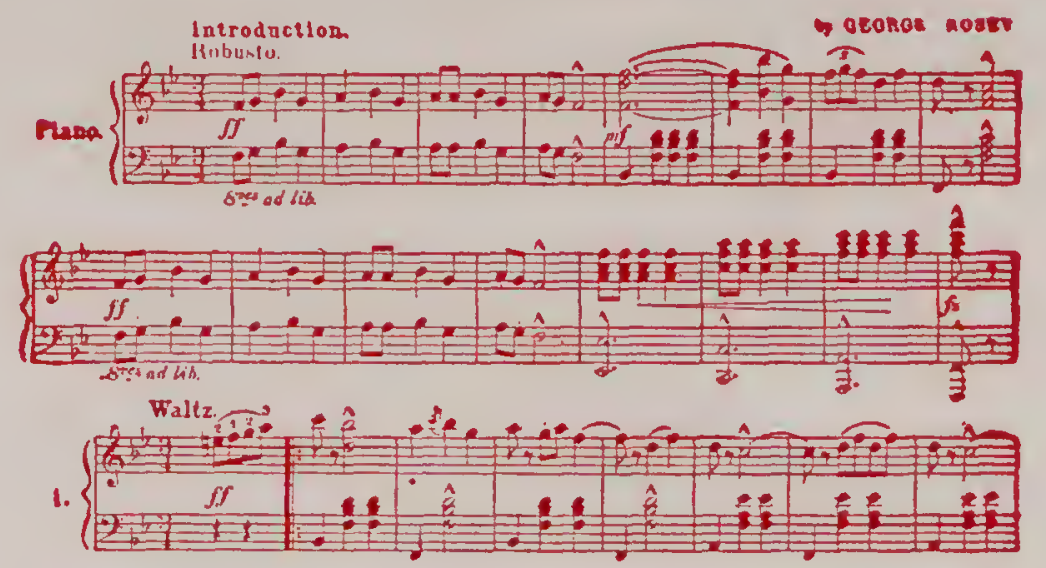

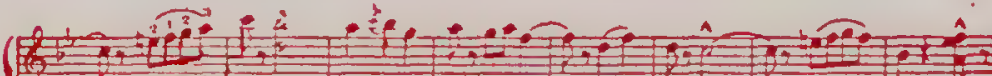

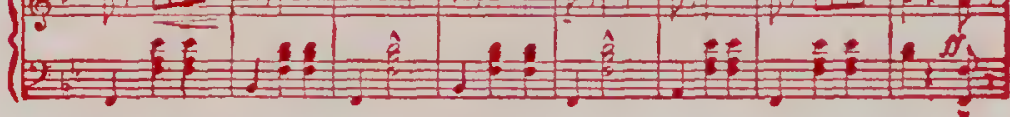

(5)

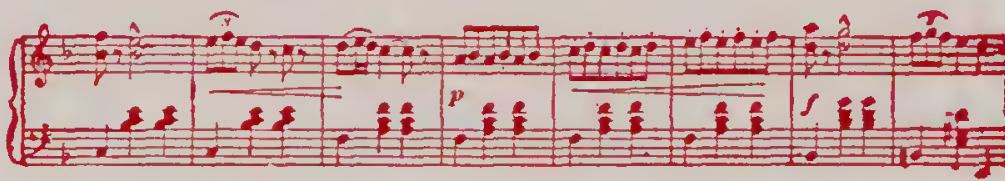

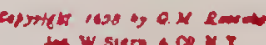

Georße Rosey's Beauliful Concerl Wallz PLAYEd AT ALL THEATRES

Rendez.Vous.Valse

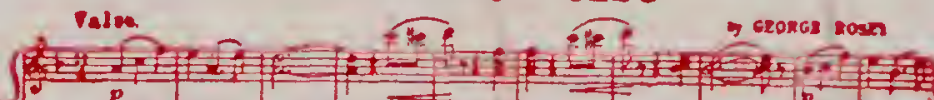

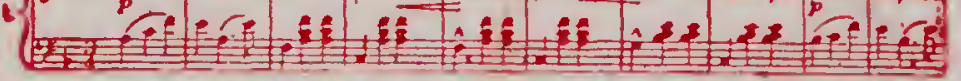

M

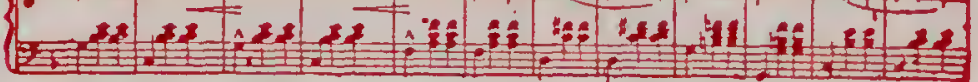

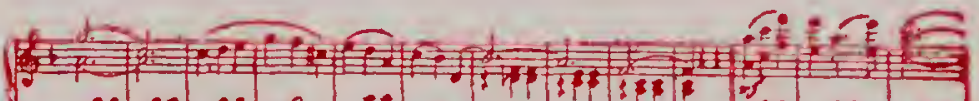

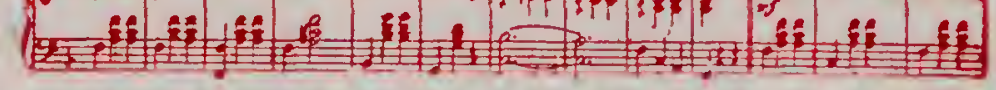

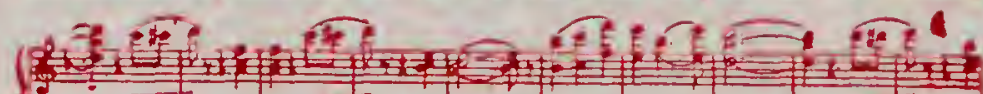

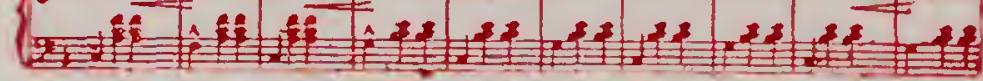

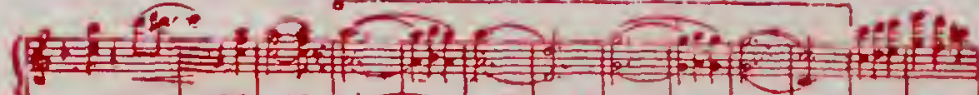

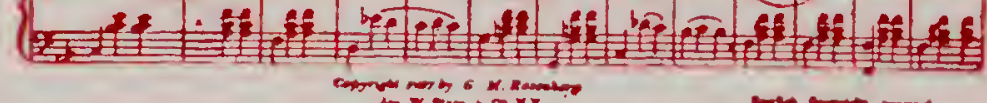

George Rosey's Famous Waltz

Played by every Leadiny Orrhesire and on My Lady Love

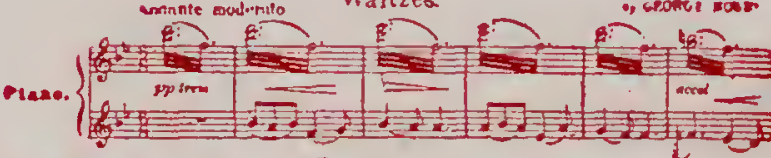
4 4.

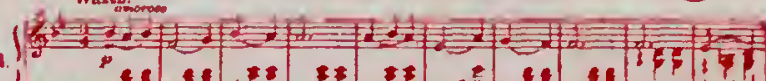

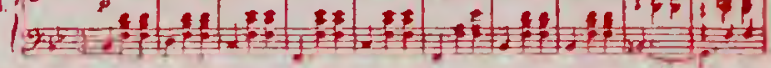

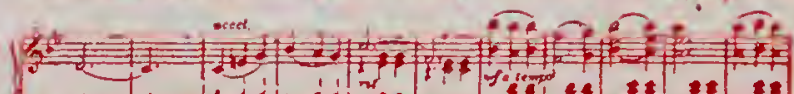

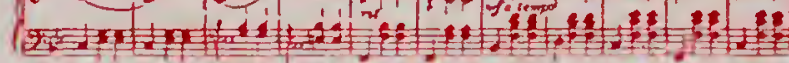

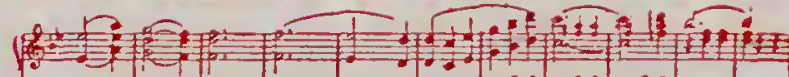

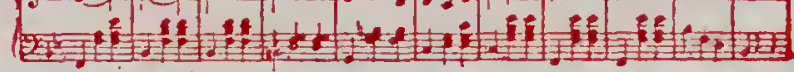

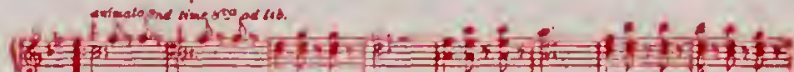
(2,3)

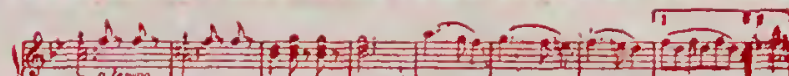

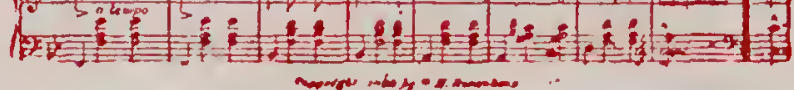

GOROE ROSEY'S NEW WINNEA A RAG-TIME SKEDADDLE

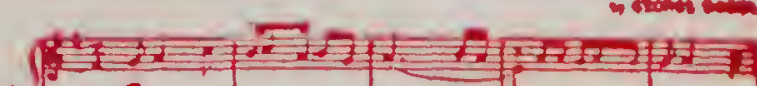

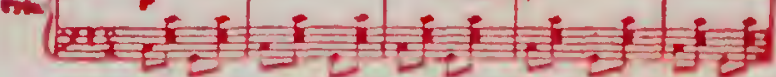

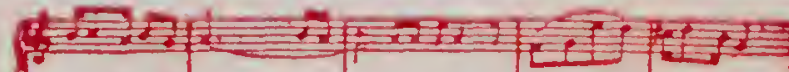

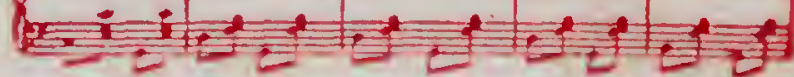

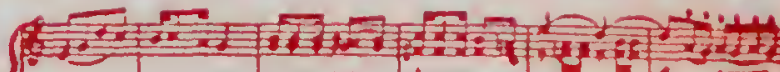
(25ín

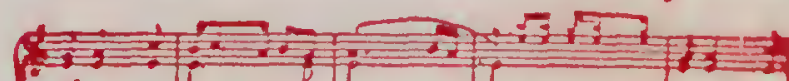
E:

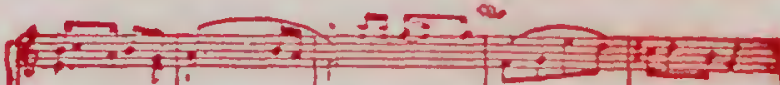

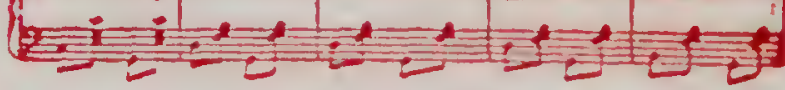

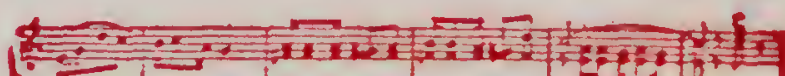

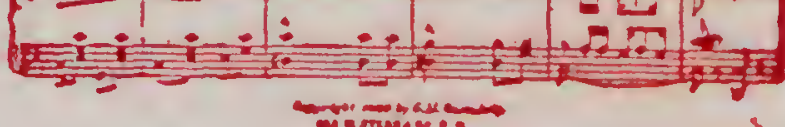

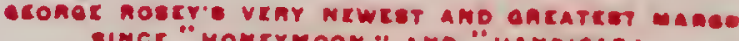
A NIGHT OFF

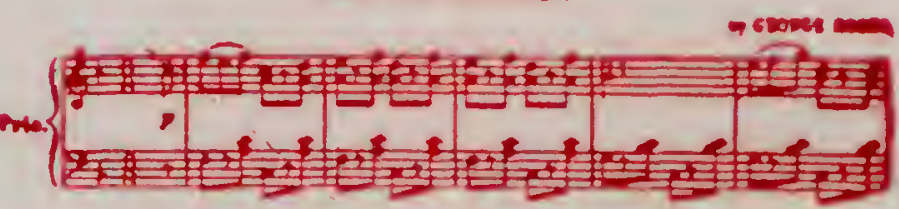

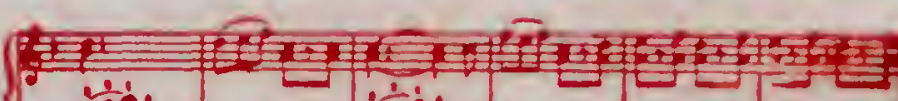

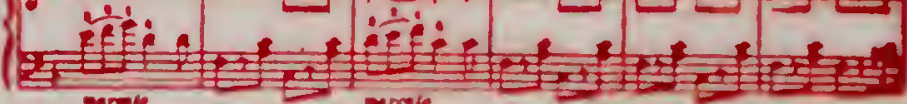
(4)

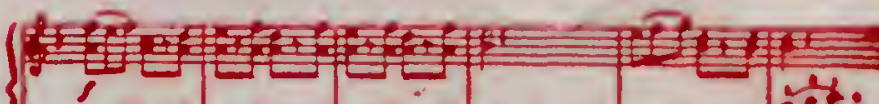
年

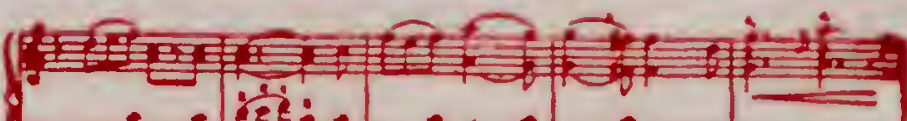

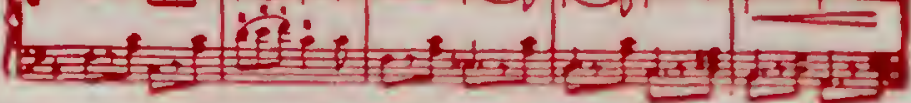

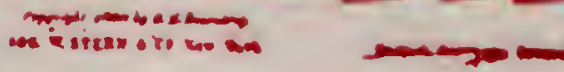

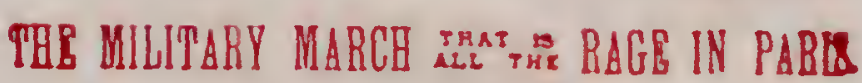
FOLIES.BERGERE YARCB $\rightarrow$ TWO STEP

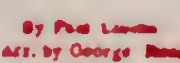

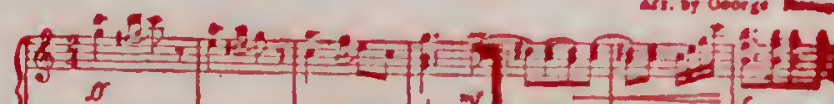

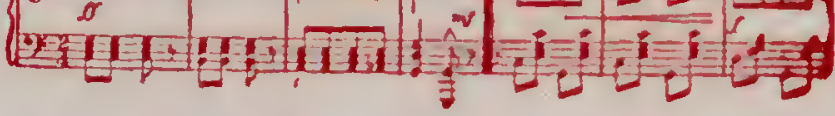

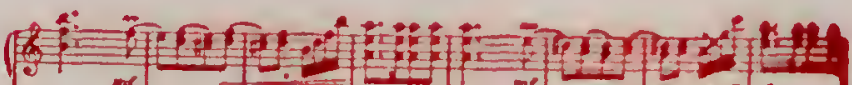

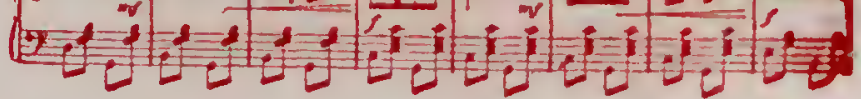

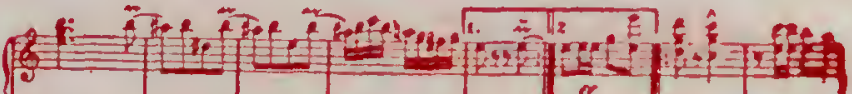

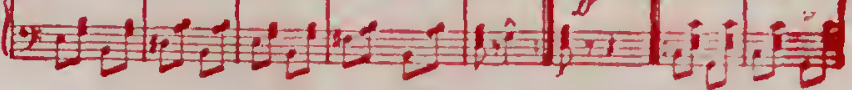

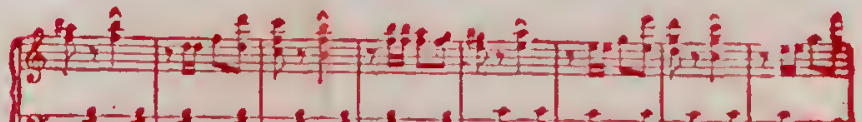

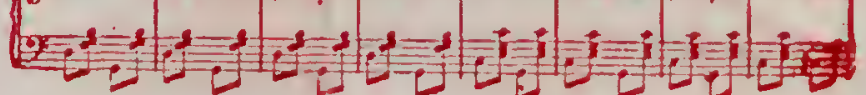

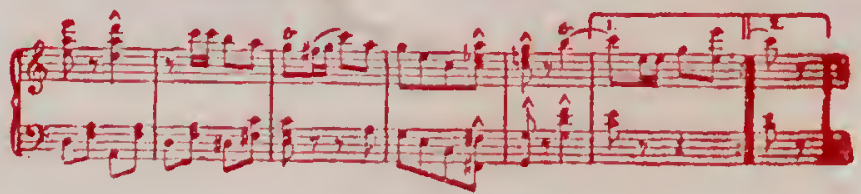

COMPIFTF COPI S CAN BF. OBTAINED AT MIL MUSIC SIUKHS. PRICE 5O CENTS. 